P186 (continued)

Interview Survey and Propensity Score Matching, we create matched intervention and comparison groups of adults age 60 and over. The treatment group $(\mathrm{n}=1,717)$ comprises SNAP participants, and the comparison group $(n=3,329)$ includes eligible non-participants.

Outcome Measures and Analysis: The outcome is a binary variable for engaging in CRN, and the treatment is SNAP participation. Probit regression models are used to determine the impact of SNAP on CRN while controlling for a wide array of sociodemographic and health-related factors.

Results: SNAP participants are 3.1 percentage points less likely to engage in CRN than eligible non-participants $(p=0.06)$. This effect is magnified for the subgroup of older adult SNAP participants at-risk of hunger, who were 5.8 percentage points less likely to engage in CRN than their counterparts $(\mathrm{p}=0.04)$.

Conclusions and Implications: Approximately 42 percent of eligible older adults participate in SNAP. Health systems and payers have a vested interest in connecting low-income older adults with food assistance programs given the direct connection between food insecurity and poor health outcomes, and now strong evidence that SNAP participation can help older adults afford their prescription medications.

Funding: None

\section{P187 SNAP-Eligible Families Encounter Multiple Economic, Social, and Environmental Barriers to Fruit and Vegetable Consumption}

Kara Golis, BS, RD, golis.5@buckeyemail.osu.edu, The Ohio State University, 1787 Neil Avenue, Columbus, $\mathrm{OH}$ 43210; Elizabeth Hustead, MS, The Ohio State University; Ana Claudia Zubieta, PhD; Brian Butler, MPH;

Joyce Counihan, MA, CPS Baobab; Julie Kennel, PhD, LDN, $R D$, The Ohio State University

Objective: Low-income households devote fewer dollars per person to the purchase of fruits and vegetables (FV). Eating FV in place of more energy-dense foods is associated with body weight management and reduced risk for many chronic diseases. Social marketing (SM) interventions have shown to influence health behavior, thus, Ohio SNAP-Ed is developing a SM campaign to increase FV demand and consumption among the target population. This study's purpose was to explore dietary patterns and motivations/barriers to FV consumption among Ohio SNAP-eligible families to inform campaign development.

Study Design, Setting, Participants: Cross-sectional, mixed methods approach using focus groups and an online survey. Participants were primarily SNAP-eligible adults at or below $185 \%$ poverty with children living in the home.

Outcome Measures and Analysis: Family characteristics (e.g., income, ethnicity, number of children in household); behaviors related to purchasing, preparation, and consumption of FV; motivators and barriers associated with eating FV. Focus groups were recorded, transcribed, and analyzed using thematic analysis techniques in NVivo. Three trained researchers coded survey responses and distilled collective findings into thematic observations.

Results: Thirteen themes emerged, including the prevailing influence of cost, family member taste preferences, and shelf life on food purchases. Nearly half (48.1\%) of the target population felt that they eat "enough" fruits and vegetables, despite reporting intake levels well below USDA recommendations.

Conclusions and Implications: The economic, social, and environmental barriers to FV consumption identified by Ohio SNAP-eligible adults justify a need for innovative SM strategies to enhance existing SNAP-Ed programs. Consideration of such barriers will ultimately guide and strengthen the design of a SM campaign.

Funding: Supplemental Nutrition Assistance Program Education

\section{P188 Summarizing Common Achievements, Challenges, and Lessons Learned of USDA Farm to School Grantees}

Lauryn Hong, BA, USDA Food and Nutrition Service Office of Community Food Systems; Matthew Benson, PhD, matthew.benson@fns.usda.gov, USDA Food and Nutrition Service Office of Community Food Systems, 3101 Park Center Drive, Alexandria, VA 22302; Matthew Russell, MNO, USDA Food and Nutrition Service Office of Community Food Systems; Andrew Powers, PEER Associates; Mieka Sanderson, MPH, USDA Food and Nutrition Service Office of Community Food Systems

Objective: Farm to school programs involve efforts to bring locally produced foods into school cafeterias; facilitate hands-on learning activities such as school gardening, farm visits, and culinary classes; and integrate food-related education into standards-based classroom curriculum. This research summarizes common achievements, challenges, and lessons learned of USDA Farm to School Program grantees.

Study Design, Setting, Participants, Intervention: USDA's Office of Community Food Systems (OCFS) annually funds school district involvement in farm to school through its Farm to School Grant Program. From 2013 to 2016, USDA funded 300 projects in all 50 states including 152 school districts or charter school programs in 42 states and territories.

Outcome Measures and Analysis: Staff in OCFS performed a qualitative analysis on final reports submitted by 83 school districts that received a USDA Farm to School grant between 2013 and 2015. Themes were grouped by achievements, challenges, and lessons learned using USDA's farm to school curriculum which includes 12 separate planning and implementation modules. 